\title{
Comet McNaught C/2006 P1: observation of the sodium emission by the solar telescope THEMIS
}

\author{
F. Leblanc ${ }^{1,2}$, M. Fulle ${ }^{2}$, A. López Ariste ${ }^{3}$, G. Cremonese $^{4}$, A. Doressoundiram ${ }^{5}$, A. Sainz Dalda ${ }^{6,7}$, and B. Gelly ${ }^{3}$ \\ 1 Service d'Aéronomie, Université Versailles Saint Quentin, CNRS, France \\ e-mail: francois.leblanc@aerov.jussieu.fr \\ 2 INAF - Osservatorio Astronomico di Trieste, Italy \\ 3 THEMIS - CNRS UPS 853 C/ Via Lactea s/n, 38200 La Laguna, Spain \\ 4 INAF - Osservatorio Astronomico di Padova, Italy \\ 5 Observatoire de Paris-Meudon, LESIA, France \\ 6 THEMIS, S.L. C/ Via Lactea s/n, 38200 La Laguna, Spain \\ 7 IAC, C/ via Lactea s/n, 38200 La Laguna, Spain
}

Received 4 October 2007 / Accepted 31 January 2008

\section{ABSTRACT}

\begin{abstract}
Comet McNaught C/2006 P1 was the brightest comet of the last forty years when reaching its perihelion at an heliocentric distance of $0.17 \mathrm{AU}$. Two days before this perihelion, at an heliocentric distance of $0.2 \mathrm{AU}$, Themis, a French-Italian solar telescope in the Canary Islands, Spain, observed the Comet sodium emission of McNaught.

The measured maximum sodium brightness of the D2 emission line peaked at 900 Mega-Rayleigh. The spatial distribution of the sodium emission with respect to the nucleus of the comet is in agreement with previous observations. It displays a clear sunwardtailward asymmetry that suggests a dichotomy of the sodium sources between a source close to the nucleus and an extended source most probably corresponding to the dust tail. The spatial distribution along the slit of the width and speed of the Doppler Na distribution also suggests such a dichotomy. The sodium ejection rate inferred from this observation agrees with the value of the ejection rate extrapolated from comet Hale-Bopp, taking into account the heliocentric distance of comet McNaught and its significantly larger dust release.

If we suppose a similar concentration of sodium atoms in both comets, this observation suggests that the sodium ejection rate from comets McNaught and Hale-Bopp is proportional to the solar flux. Therefore the most probable ejection mechanisms are photosputtering, solar wind sputtering, or cometary ion sputtering, and not thermal desorption.
\end{abstract}

Key words. comets: general - instrumentation: spectrographs - solar system: general

\section{Introduction}

Even after one century of observing cometary sodium emission, the sources of the sodium atoms, the mechanisms by which sodium is released from the nucleus and from the dust grains and even the number of tails formed by the sodium atoms, are all still debated questions. The last set of observations of cometary sodium was obtained for comet Hale-Bopp C/1995 O1 in 1997 (Cremonese et al. 1997; Barker et al. 1998; Wilson et al. 1998; Brown et al. 1998; Rauer et al. 1998; Arpigny et al. 1998; Furusho et al. 2005). Cremonese \& Fulle (1997) concluded that comet Hale Bopp had four distinct tails: an ion tail along the direction of the solar wind, a dust tail, and two sodium tails. The first of the sodium tails is a diffuse tail associated to the dust tail and most probably produced by the release of the sodium atoms from the dust grains (Wilson et al. 1998; Cremonese \& Fulle 1997). The second tail, much narrower, is originally from a region close to the nucleus of the comet and is driven by the solar radiation pressure in a quasi anti-sunward direction (Cremonese et al. 1997).

Thanks to spectroscopic observation of the coma, Arpigny et al. (1998) and Rauer et al. (1998) concluded that the inner coma sodium distribution (the origin of the narrow tail) cannot be explained by an origin solely in the nucleus but should be associated with mechanisms releasing the sodium atoms from dust grains just that had been ejected from the nucleus or from a molecular parent. Different mechanisms of ejection from the dust grains, such as photo-sputtering or solar wind sputtering, have been suggested; but according to Ip and Jorda (1998), these two mechanisms should be not efficient enough to explain the observed ejection rate from comet Hale-Bopp. Therefore, other mechanisms have been suggested, such as the impact of very small dust grains (smaller than $100 \AA$ ) on the dust grains (Ip \& Jorda 1998) or an ionic parent for the Na observed emission (Combi et al. 1997). The latter solution has been however rejected by Brown et al. (1998), who found that such a possibility would produce sodium velocities that contradict the observed ones and could not explain a sodium tail that is spatially distinct from the ionic tail. The sodium distribution close to the nucleus displays a sunward/tailward asymmetry. A secondary sunward maxima of the sodium emission (Rauer et al. 1998) was observed and explained by the preferential release of the dust grains, origin of the observed sodium atoms, from the Sun side of the nucleus.

The sodium production therefore does not agree with a source solely from the nucleus, but most probably is associated with an extended source like the dust grains (Arpigny et al. 1998). The overall rate of ejected sodium from comet Hale-Bopp 
Table 1. 10 January 2007 observation. The exposure time was $20 \times 2 \mathrm{~s}$.

\begin{tabular}{cccccccc}
\hline \hline $\begin{array}{c}\text { Slit } \\
\mathrm{N}^{\circ}\end{array}$ & UT & Position & $\begin{array}{c}\text { Comet } \\
\text { Sun velocity } \\
\left(\mathrm{km} \mathrm{s}^{-1}\right)\end{array}$ & $\begin{array}{c}\text { Comet } \\
\text { Earth velocity } \\
\left(\mathrm{km} \mathrm{s}^{-1}\right)\end{array}$ & $\begin{array}{c}\mathrm{D} 2 \\
\text {-factor } \\
\left(\mathrm{s}^{-1}\right)\end{array}$ & $\begin{array}{c}\text { Maximum } \\
\text { Continuum } \\
(\mathrm{kR} / \AA)\end{array}$ & $\begin{array}{c}\text { Sky } \\
(\mathrm{kR} / \AA)\end{array}$ \\
\hline 3 & 15.59 .53 & $\begin{array}{c}\text { Centred on peak } \\
\text { of Na emission }\end{array}$ & -32.05 & -74.62 & 242.6 & 887 & 13076 \\
6 & 16.28 .59 & $\begin{array}{c}\text { Centred on peak } \\
\text { of Na emission } \\
7\end{array}$ & -31.83 & -74.51 & 242.8 & 257 & 14015 \\
\hline & 17.53 .09 & $\begin{array}{c}\text { Centred on peak } \\
\text { of Continuum+sky } \\
\text { Emission }\end{array}$ & -31.19 & -74.21 & 243.6 & 2403 & 231544 \\
\hline
\end{tabular}

suggests that most of the sodium of the comet remains confined in the cometary dust.

More recently, comet McNaught reached heliocentric distances smaller than $0.2 \mathrm{AU}$ and became the brightest comet of the last 40 years, the perihelion $\operatorname{Af} \rho$ of comet McNaught being larger by a factor 10 than that of comet Hale-Bopp at perihelion (Sostero 2007). Fulle et al. (2007) used the observation by STEREO/Heliospheric Imager of this comet, and for the first time identified another atomic tail constituted of iron atoms lost by comet McNaught with a rate of $10^{30} \mathrm{~s}^{-1}$. The HCN emission line observation also suggested a huge water loss as it was ejected with a velocity of $1.5 \mathrm{~km} \mathrm{~s}^{-1}$ at the nucleus (Biver $\mathrm{N}$. personal communication), in agreement with Fulle et al. (2007).

In this paper, we present and analyse observations of the sodium emission of comet McNaught that were obtained on January 10, 2007, using THEMIS, a French-Italian solar telescope in the Canary Islands, Spain.

\section{Data}

THEMIS (Rayrole et al. 1998; Loṕez Ariste et al. 2000) is a French-Italian solar telescope based on the Canary Island of Tenerife, Spain (Latitude: North 28 $18^{\prime} 12.42^{\prime \prime}$, Longitude: West $16^{\circ} 30^{\prime} 32.04^{\prime \prime}$, Elevation: $2429 \mathrm{~m}$ ) with a $90-\mathrm{cm}$ primary mirror (with an obscuration of $400 \mathrm{~mm}$ ) and a $15.04 \mathrm{~m}$ focal length. It is the first solar telescope on an alt-az mounting, a characteristic that allows pointing not just to the Sun, but to any point in the celestial sphere. For this particular observation, the telescope was used in a spectroscopic configuration with only one camera centered on the D2 emission line at $5889 \AA$ with a band width of less than $6 \AA$. The camera is composed of 512 pixels in the dispersion direction and 512 in the spatial direction. The associated dispersion is $\sim 0.01 \AA /$ pixel that is $0.6 \mathrm{~km} \mathrm{~s}^{-1}$ at $\mathrm{Na} \mathrm{D} 2$ wavelength. The slit size was equal to $\sim 2^{\prime}$ that is $0.25^{\prime \prime}$ per pixel and was $1.5^{\prime \prime}$ large (corresponding to $80 \mathrm{~m} \AA$ of spectral resolution).

The flat field was done with sky observations taken far from comet McNaught emission. Since these observations were performed during daylight, the sky-reflected light provided a relatively good equivalent constant continuum within the considered band width. This method worked properly for slit positions 3 and 6 but did not succeed for slit 7 , so that no flat field was possible for this last slit position (Table 1). The wavelength calibration was based on the identification of the main telluric lines within the band width. The absolute calibration of the observation was based on a dedicated observation of Venus. The global uncertainties are of less than one pixel for the spectral calibration and about $20 \%$ for the absolute calibration (based on Venus observation and ephemeris magnitude).

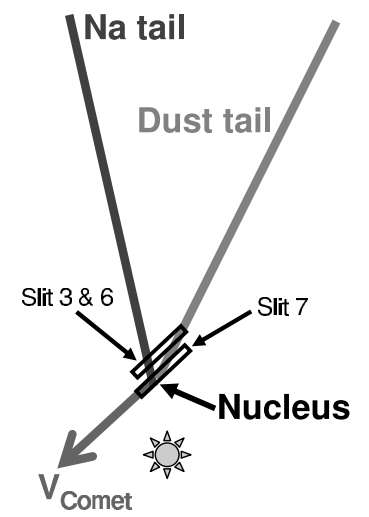

Fig. 1. Cartoon of the observation done by Themis 10 January 2007. The three positions during that observation, namely, slit positions 3,6 , and 7 are displayed in this diagram. The orientations of the sodium tail and of the dust tail have been calculated from the syndyne model (Fulle 2004).

Unfortunately, only one day of observation, 10 January 2007 , with only three individual exposures were obtained because of bad weather conditions. The UT time and the Doppler velocities of Comet McNaught compared to the Sun and to the Earth are provided in Table 1. On that day, the comet was at a heliocentric distance of $0.192 \mathrm{AU}$ and at a geocentric distance of $0.93 \mathrm{AU}$. The perihelion of the comet occurred on 12 January 2007 at 12.8 UT at a distance of 0.171 AU to the Sun. On 10 January 2007, comet McNaught was observable with a phase angle of 100 degrees.

The slit position compared to the Comet motion was controlled by monitoring the telescope field of view and by manually moving the slit position. The slit was placed so that the proper motion of the comet was parallel to the slit. The estimated error on the angle between the slit and the exact velocity direction of comet McNaught was estimated to be less than $10^{\circ}$. For slit positions 3 and 6 , the slit was placed parallel to the proper motion of the comet and centred on the maximum of the sodium emission, whereas for slit 7 the slit was placed on the maximum of the continuum (localized by moving the slit around the comet manually). Its distance to the nucleus was estimated to be of less than $30^{\prime \prime}$. The seeing value was evaluated to be between $1^{\prime \prime}$ and $2^{\prime \prime}$.

The positions of the slit during the observation are displayed in Fig. 1. We also indicate in this sketch the position of the Sun, the dust tail, and the $\mathrm{Na}$ tail. The approximate orientations of these two tails were calculated based on the syndyne analysis (Fulle 2004). 

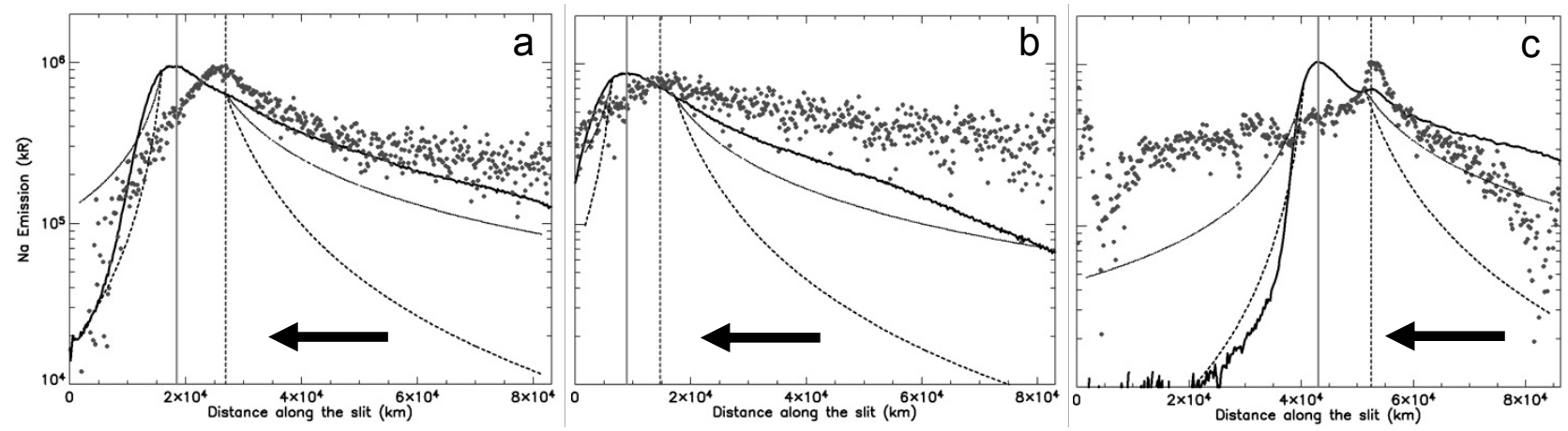

Fig. 2. Sodium integrated emission vs. distance along the slit (solid line) and continuum intensity rescaled to the sodium emission intensity for convenience (dots). Also plotted are two lines representing a variation in the emission following a $1 / r$ variation from the peak in emission (solid gray lines) and following a $1 / r^{2}$ variation (dotted lines). The vertical solid line corresponds to the position of the peak in sodium emission, whereas the dotted vertical line corresponds to the peak in the continuum emission. Panel a) slit position 3. Panel b) slit position 6. Panel c) slit position 7. The arrow in each panel indicates the velocity direction of the comet.

\section{Analysis}

We analyzed the sodium emission line for each pixel along the slit. The continuum+sky contribution to the sodium emission line was estimated from two spectral domains on each side of the comet's sodium emission line. The sky contribution was also roughly estimated from the part of the slit placed before the comet in the velocity direction of the comet. At this particular position, the dust density and therefore the scattered light from the dust should be negligible. The sodium emission was integrated after subtraction of this continuum+sky contribution. For the three slit positions, Fig. 2 displays the continuum and the emission sodium brightness vs. distance along the slit. The scale of the continuum intensity was artificially fitted to the scale of the sodium emission. The continuum of the comet cannot be evaluated accurately because we were not able to subtract the sky contribution properly, and because of the limited quality of the flat field, in particular at the edge of the slit. Absolute values of the continuum and of the sky intensity are provided in Table 1 for comparison. From Fig. 2, it is clear that for slit position 6 the nucleus was almost at the edge of the slit so that the sky contribution was probably overestimated. From the values of the continuum displayed in Table 1, slit position 7 was clearly placed closer to the nucleus of the comet than slit positions 3 and 6 . The sky contribution to these measurements changed from slit positions 3 and 6 to slit position 7 by up to a factor 20 , which we attributed to cloud-reflected light during slit position 7 . The maximum value of the continuum for slit positions 3 and 6 (knowing the uncertainty on the sky subtraction for slit position 6) suggests that these two positions were relatively close to each other.

The other main features of Fig. 2 are:

- The maximum of the emission sodium intensity is very similar for all the slit positions, namely around 900 MRayleigh.

- The signal then decreases in the opposite direction of the comet velocity, roughly following a $1 / r$ variation in intensity and along the comet velocity following a $1 / r^{2}$ variation where $r$ is the distance to the peak in intensity.

- The position of the peak in the sodium emission is not at the same position as the observed maximum continuum. The observed localized bump in the continuum (Fig. 2) cannot be caused by a localized variation in the sky intensity along the slit. These two peaks are separated by around $10000 \mathrm{~km}$. Supposing that the continuum peak is associated with the comet McNaught nucleus on slit position 7, the sodium emission peak therefore occurred before the comet nucleus in the
velocity/Sunward direction by a distance of more than $13^{\prime \prime}$, which is significantly more than the seeing value.

From the integrated emission intensity, it is possible to infer the optical thickness of this emission by extrapolating a Brown \& Yung (1976) calculation applied to the sodium at Io to the McNaught heliocentric distance. By using Eq. (17) in Brown \& Yung (1976) and a temperature of $700 \mathrm{~K}$ estimated for the dust at this heliocentric distance (Fulle et al. 2007), the optical thickness of the D2 line is equal to one when the column density is equal to $1.2 \times 10^{11} \mathrm{Na} / \mathrm{cm}^{2}$. However, since the apparent velocity distribution of the $\mathrm{Na}$ atoms will also depend on both the velocity distribution of the dust ejected at $1.5 \mathrm{~km} \mathrm{~s}^{-1}$ from the nucleus (Fulle et al. 2007), which corresponds to $3000 \mathrm{~K}$, and on the ejection mechanism from the dust, a better approximation of the sodium apparent temperature should be at least a temperature of $3000 \mathrm{~K}$. At such a temperature, the optical thickness is equal to one when the column density is equal to $2.5 \times 10^{11} \mathrm{Na} / \mathrm{cm}^{2}$. Therefore, using the Brown \& Yung (1976) relation between the emission intensity of the D2 line and Na column density (their Fig. 6a) at Io and rescaling it at comet McNaught's heliocentric distance, the optical thickness of the emission is more than 10 for comet McNaught sodium emission intensities at the peak of 900 MRayleigh and is greater than one along most of the slit.

A Gaussian distribution is a good approximation of the sodium emission line measured by Themis. At all positions along the slit, the measured spectra display a small but clear deficit, in comparison with the Gaussian distribution, in particles with a positive Doppler velocity greater than $4 \mathrm{~km} \mathrm{~s}^{-1}$ with respect to the Doppler velocity of comet McNaught nucleus (which was estimated from ephemeris). A positive Doppler velocity corresponds to particles moving away from the observer and towards the Sun. At that time, solar pressure was pushing the observed atoms towards the observer (in the blue) so that this deficit must be due to the solar pressure.

Using the Gaussian fit, we deduced the Doppler speed of the emission spectrum (the barycentre of the Gaussian distribution) compared to the nucleus with a $\sim 0.1 \mathrm{~km} \mathrm{~s}^{-1}$ precision (Fig. 3). The Doppler velocity is negative compared to the nucleus Doppler velocity as expected for atoms pushed by the solar radiation pressure towards the observer (in agreement with the Barker et al. 1998, observation of Hale-Bopp Na emission). For slit positions 3 and 6 , the profiles are fairly similar, and they display two distinct peaks (panels a and b). The regions between these two peaks correspond to the region where the sodium emission has an optical thickness much greater than one. The 

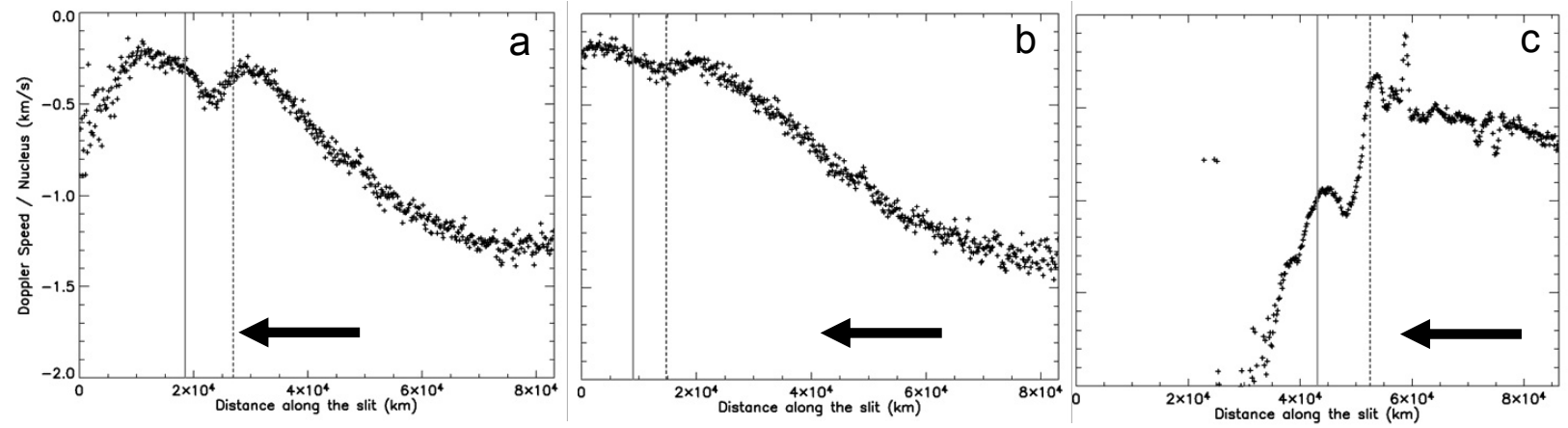

Fig. 3. Relative Doppler speed of the sodium atoms compared to Earth in $\mathrm{km} \mathrm{s}^{-1}$ vs. distance along the slit in the frame of comet McNaught. Panel a) slit position 3. Panel b) slit position 6. Panel c) slit position 7. The vertical solid line corresponds to the position of the peak in sodium emission, whereas the dotted vertical line corresponds to the peak in the continuum emission. The arrow in each panel indicates the velocity direction of the comet.
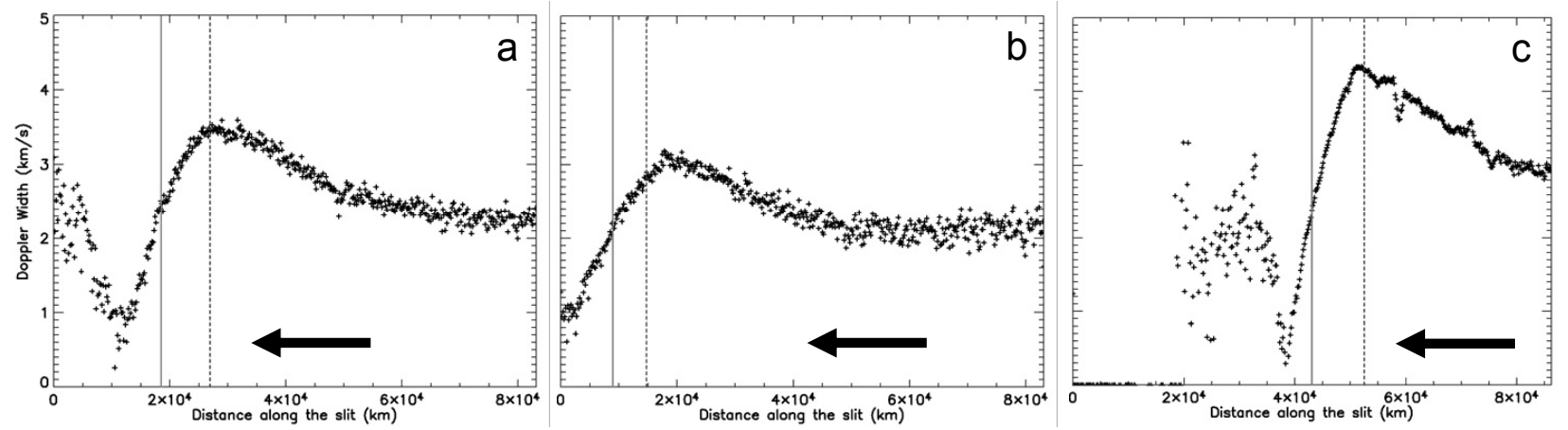

Fig. 4. Doppler width of the sodium atoms in $\mathrm{km} \mathrm{s}^{-1}$ vs. distance along the slit in the frame of comet McNaught (full half maximum width of the Gaussian function fitted on the spectrum in each pixel of the slit). Panel a) slit position 3. Panel b) slit position 6. Panel c) slit position 7. The vertical solid line corresponds to the position of the peak in sodium emission and the dotted vertical line to the peak in the continuum emission. The arrow in each panel indicates the velocity direction of the comet.

minimum between the two peaks may be produced by the thickness of the emission along the line of sight, shadowing the atoms moving away from the observer that have positive Doppler velocity. If the emission was optically thin everywhere, we should have observed a maximum Doppler velocity where the peak in sodium ejection occurred within the slit and a decrease away from it due to the phase angle between the line of sight and the mean ejection direction. In the case of slit position 7 (panel c), the peak in the Doppler velocity at $-1 \mathrm{~km} \mathrm{~s}^{-1}$ corresponding to the peak in the $\mathrm{Na}$ emission (at the position $40000 \mathrm{~km}$ along the slit) is most probably associated to the part of the $\mathrm{Na}$ population visible from the Earth, which is essentially those atoms moving towards the observer (the optical thickness is more than 10 at this position). Therefore, $1 \mathrm{~km} \mathrm{~s}^{-1}$ is also close to the velocity (with respect to the nucleus of the comet) of the sodium atoms when ejected from the dust grains. As a consequence, such a velocity should be very close to the dust-grain velocity when ejected from the nucleus. This value of $1 \mathrm{~km} \mathrm{~s}^{-1}$ is actually less than the observed velocity of $1.5 \mathrm{~km} \mathrm{~s}^{-1}$ of the HCN emission line as observed by Biver et al. (private communication, 2007). A lower velocity is probably due to the contribution of the sodium atoms moving away from the observer along the line of sight or simply to the accuracy of this measurement. The hole at the $50000 \mathrm{~km}$ position, panel $\mathrm{c}$ is due to the lack of flat field correction for slit position 7.

From the Gaussian fit, it is also possible to derive the Doppler width of the spectra (the FWHM) knowing the spectral dispersion due to the spectrograph. The results of the measured Doppler width along the slit are displayed in Fig. 4. A peak corresponding to a Doppler width of 3 to $4 \mathrm{~km} \mathrm{~s}^{-1}$ is observed very close to the maximum of the continuum whereas the Doppler width is reduced to $2 \mathrm{~km} \mathrm{~s}^{-1}$ at the position of the maximum of the sodium emission. Actually the peak in Doppler width only appears slightly behind the maximum of continuum in the case of slit 6. This good correlation between maximum of Doppler width and maximum of continuum, in particular in the case of slit 7 where the slit was the closest to the nucleus, therefore suggests that the closest point to the nucleus within the slit might correspond to the peak in the Doppler width. Similar values were reported for Hale-Bopp by Barker et al. (1998). These authors observed a first core population of $\mathrm{Na}$ atoms with Doppler width between 1.6 and $2.7 \mathrm{~km} \mathrm{~s}^{-1}$ and a secondary minor population with Doppler width between 1.9 and $6.2 \mathrm{~km} \mathrm{~s}^{-1}$ and globally shifted by $\sim 3 \mathrm{~km} \mathrm{~s}^{-1}$ compared to the core population. These authors concluded that the $2 \mathrm{~km} \mathrm{~s}^{-1}$ Doppler width population is probably produced close to the slit and that the secondary population is probably the core population accelerated in the antisolar direction by the solar radiation pressure. In our case, we do observe two different populations with different Doppler width and similar respective intensity, but we do not observe the same shift in Doppler speed as displayed in Fig. 3 even if, in particular in the case of slit position 7, a small Doppler speed shift between these two populations can be seen. The most probable explanation for this discrepancy is due to the optical thickness 


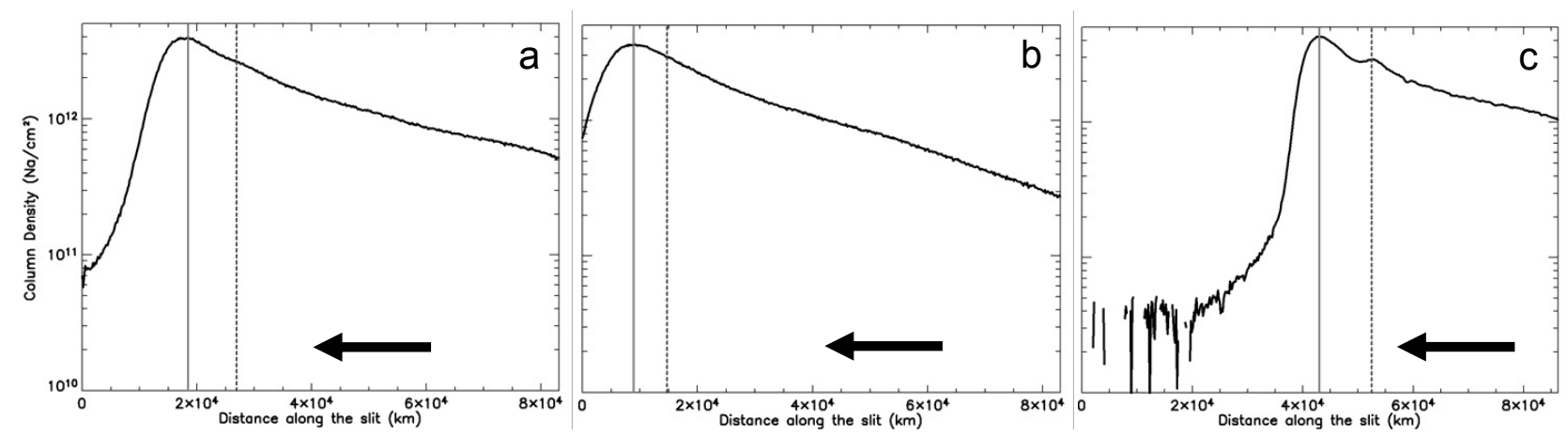

Fig. 5. Sodium column density $\left(\mathrm{Na} / \mathrm{cm}^{2}\right)$ derived from the emission intensity using optical thin assumption. Panel a): slit position 3 . Panel b): slit position 6. Panel c): slit position 7. The vertical solid line corresponds to the position of the peak in sodium emission whereas the dotted vertical line corresponds to the peak in the continuum emission. The arrow in each panel indicates the velocity direction of the comet.

of the emission in the case of comet McNaught, whereas the Hale-Bopp emission observed by Barker et al. (1998) was optically thin.

Table 1 also provides the $g$-factor of the D2 sodium emission taking into account the Doppler of comet McNaught and the distance of comet McNaught to the Sun. These values were calculated using the high-resolution visible solar flux (Kurucz et al. 1984) and oscillator strengths for the Na D2 resonant line (Morton et al. 2003, 2004). The $g$-factor of an optically thin emission provides the proportionality between emission intensity and column density. Supposing that the emission is optically thin, we can derive the column density along each slit position. The calculated values of the column density are displayed Fig. 5 and have to be considered as a lower limit of the exact value in particular between the two vertical lines. Actually there is a small decrease in the value of the column density, as well as in the emission brightness between the two vertical lines, in particular in Fig. 5c, which might be due to an effect of the optical thickness. Using Fig. 6a of Brown \& Yung (1976) corrected for the distance to the Sun, it can be estimated that the assumption of an optically thin atmosphere should induce underestimation of the real column density by a factor up to 10 at the peak emission of $900 \mathrm{MR}$.

As can be seen in Fig. 5, the peak in the column density associated to the three slit positions is very close to being equal to $4 \times 10^{12} \mathrm{Na} / \mathrm{cm}^{2}$. The strong asymmetry in the decrease of the sodium column density on both sides of the maximum peak can be interpreted as two spatial sources of the observed sodium atoms with one source close to the nucleus (most probably from the dust grains) and a second extended source on the antivelocity/anti-Sunward side of the nucleus, probably associated with the dust grains of the dust tail as reported by Cremonese \& Fulle (1997).

It is possible to derive an approximate value for the ejection rate of the sodium atoms from comet McNaught using the observed column density. Supposing that the source of all observed sodium atoms is a point at the scale of the spatial resolution of our observation (typically the seeing value that is around $1400 \mathrm{~km}$ ), the density of sodium atoms $\rho_{\mathrm{V}}$ at any position from this point can be expressed as:

$\rho_{\mathrm{V}}\left(\mathrm{cm}^{-3}\right)=\frac{\dot{N}}{4 \Pi r^{2} V}$

where $\dot{N}$ is the ejection rate in number of $\mathrm{Na}$ atoms per second, $V$ the ejection velocity of the $\mathrm{Na}$ atoms in $\mathrm{cm} \mathrm{s}^{-1}$ and $r$ the distance to the nucleus in $\mathrm{cm}$. From Eq. (1), the relation between
$\dot{N}$ and the column density $\rho_{\mathrm{C}}$ at a distance of $R$ from the point source can be deduced as:

$\rho_{\mathrm{C}}\left(\mathrm{cm}^{-2}\right)=\frac{\dot{N}}{4 r V}$.

Using the measured column density in Fig. 5, and Eq. (2), we can estimate $\dot{N}$ along the slit as being equal to $10^{27} \mathrm{Na} / \mathrm{s}$, and it actually may be close to $10^{28} \mathrm{Na} / \mathrm{s}$ when corrected by the effect of the optical thickness of the emission. The Hale Bopp ejection rate has been estimated to be equal to $5 \times 10^{25} \mathrm{Na} / \mathrm{s}$ by Cremonese et al. (1997), between $3 \times 10^{24}$ and $5 \times 10^{25} \mathrm{Na} / \mathrm{s}$ by Rauer et al. (1998) and at $3.5 \times 10^{25} \mathrm{Na} / \mathrm{s}$ by Wilson et al. (1998) at $0.96 \mathrm{AU}$ from the Sun. If comet McNaught had the same concentration of sodium atoms as comet Hale Bop and since the photon flux at $0.191 \mathrm{AU}$ is 27 times greater than at the Hale Bopp distance to the Sun (0.96 AU), we should have found an ejection rate between $8.25 \times 10^{25}$ and $1.4 \times 10^{27} \mathrm{Na} / \mathrm{s}$ if the same dust rate was ejected from the two comets and if photo-sputtering was the dominant process in ejecting sodium atoms from the dust grains.

However, the dust Af $\rho$ parameter was observed as being at least one order larger at comet McNaught than at comet Hale Bopp (Fulle et al. 2007). Therefore the quantity of sodium released from the dust at comet McNaught also should have been one-order more than previously calculated, that is, between $8.25 \times 10^{26}$ and $1.4 \times 10^{28} \mathrm{Na} / \mathrm{s}$. The rate of $\mathrm{Na}$ ejected from comet McNaught is therefore in good agreement with the rate of comet Hale Bopp extrapolated to the comet McNaught distance to the Sun and to its dust rate. Moreover, it is possible to infer the dust density within the slit by using the maximum $2403 \mathrm{kR}$ of the continuum for slit 7. Using a solar flux at $0.191 \mathrm{AU}$ of $1.5 \times 10^{15}$ photons $/ \mathrm{cm}^{2} / \mathrm{s} / \AA$ and a dust albedo of 0.2 around the Na D2 line (from Mason et al. 2001 for a phase angle of $100^{\circ}$ ), the ratio between the continuum intensity and the solar flux is equal to less than $0.1 \%$, which suggests a low opacity of the dust. Therefore the ejection rate by photo-sputtering should not have been influenced by the dust opacity. This result contradicts the dependency of the ratio between the sodium column density and the dust column density compared to heliocentric distance as calculated by Watanabe et al. (2003) and Furusho et al. (2005). Indeed Furusho et al. (2005) estimate a dependency in $r^{-5.85 \pm 1.26}$ with $r$ the heliocentric distance. Knowing that the value of the dust $\operatorname{Af} \rho$ parameter of comet McNaught should have been at least 10 times higher than for comet Hale-Bopp and according to Eq. (2), such a dependency would imply a $1.9 \times 10^{4}$ to $80 \times 10^{4}$ higher sodium ejection rate rather than the $0.2 \times 10^{3}$ to $3 \times 10^{3}$ higher rate observed for comet McNaught than for comet Hale-Bopp. This contradiction does not rule out thermal 
desorption as a potential mechanism for the ejection of the sodium atom but strongly contradicts the conclusion that thermal desorption might be a dominant ejection process for the observed sodium atoms.

Therefore, if the same concentration of sodium is supposed in comet McNaught and comet Hale-Bopp, our observations suggest that the mechanism leading to the ejection of the observed $\mathrm{Na}$ atoms from both comets (if we suppose that it is essentially the same) has a rate proportional to the solar photon flux; that is, it is most probably related to photo-sputtering of the dust grains ejected from the nucleus. Solar wind or/and cometary ion sputtering may also contribute to the observed $\mathrm{Na}$ population. Indeed, ion sputtering is efficient if the impacting ion reaches energy higher than $100 \mathrm{eV} / \mathrm{amu}$, particularly when the impact occurs outside the cometopause. In the case of Comet 1P/Haley, the cometopause subsolar distance was estimated to be $10^{5} \mathrm{~km}$ (Ip 2004), which is significantly farther than the mean distance of $10^{4} \mathrm{~km}$ between the emission and continuum peaks reported in this work. However, considering the much stronger dust release of Comet McNaught, the order-of-magnitude higher solar wind dynamic pressure and photo-ionization frequency, the characteristics of the solar wind interaction with comet McNaught are difficult to infer without dedicated studies, so that solar wind or/and cometary ion sputtering cannot be excluded.

\section{Conclusions}

In this paper, we report the Themis observations of the sodium emission from comet McNaught, a sun-grazing comet that reached as close as 0.19 AU to the Sun. The measured maximum sodium brightness of the D2 emission line peaks at 900 Mega-Rayleigh. The measured D2 emission displays the spatial dichotomy between (i) a spatially localized source peaking at a few tens of thousand kilometres in the velocity direction and Sunward from the nucleus of comet McNaught; and (ii) an extended source most probably associated to the dust tail in the anti-velocity direction. This observation displays a clearly dominant peak in the emission associated to the spatially localized source. This feature contradicts the Rauer et al. (1998) sodium emission observation of comet Hale-Bopp. They reported a peak in the Sunward direction less intense than the peak in the sodium emission centered on the Hale-Bopp nucleus. The deduced global sodium ejection rate appears to be similar to the comet Hale-Bopp ejection rate, taking into account the difference in heliocentric distance and the one-order higher dust ejection rate at comet McNaught. Such an ejection rate suggests that the main mechanism leading to the ejection of sodium atoms from the dust grain of comets McNaught and Hale-Bopp is roughly proportional to the solar flux. In another way, this observation suggests that the observed sodium atoms are probably ejected by a process, that is photo-sputtering, solar wind sputtering, or cometary ion sputtering and not thermal desorption.

Acknowledgements. We thank the Themis technical team for their great help with this observation

\section{References}

Arpigny, C., Rauer H., Manfroid, J., et al. 1998, A\&A, 334, L53

Barker, E. S., Cochran, A. L., \& Cochran, W. D. 1997, Earth, Moon and Planets, 78, 93

Brown, R. A., \& Yung, Y. L. 1976, Io, its atmosphere and optical emissions, in Jupiter, ed. T. Gehrels (University of Arizona Press), 1102

Brown, M. E., Bouchez, A. H., Spinrad, H., \& Misch, A. 1998, Icarus, 134, 228 Combi, M. R., DiSanti, M. A., \& Fink, U. 1997, Icarus, 130, 336

Cremonese, G., \& Fulle, M. 1997, Earth, Moon, and Planets, 79, 1/3, 209

Cremonese, G., Boehnhardt, H., Crovisier, J., et al. 1997, ApJ, 490, L199

Fulle, M. 2004, Motion of Cometary dust, in Comets II, ed. M. C. Festou, H. U. Keller, \& H. A. Weaver (Tucson: Univ. Arizona Press), 565

Fulle, M., Leblanc, F., Harrison, R. A., et al. 2007, ApJ, 661, L93

Furusho, R., Kawakita, H., Fujii, M., \& Watanabe, J.-I. 2005, ApJ, 618, L543

Ip, W.-H., \& Jorda, L. 1998, ApJ, 496, L47

Ip, W.-H. 2004, Comets II, ed. M. C. Festou, H. U. Keller, \& H. A. Weaver (Tucson: University of Arizona Press) 745, 605

Kurucz, I. F., Brault, J., \& Testerman, L. 1984, National Solar Observatory Atlas No. 1, NSO/Kitt Peak FTS data used here were produced by NSF/NOAO López Ariste, A., Rayrole, J., \& Semel, M. 2000, A\&A, 142, 137

Mason, C. G., Gehrz, R. D., Jones, T. J., et al. 2001, ApJ, 549, 635

Morton, D. C. 2003, ApJS, 149, 205

Morton, D. C. 2004, ApJS, 151, 403

Rauer, H., Arpigny, C., Manfroid, J., Cremonese, G., \& Lemme, C. 1998, A\&A, 334, L61

Rayrole, J., Mein, P., \& Schmieder, B. 1998, First Observations with THEMIS, in Three-Dimensional Structure of Solar Active Regions, ed. C. E. Alissandrakis, \& B. Schmieder, ASP Conf. Ser., 155, 260

Sostero, G. 2007, Cometary Data Archive for Amateur Astronomers, http:// cara.uai.it

Watanabe, J.-I., Kawakita, H., Furusho, R., \& Fujii, M. 2003, ApJ, 585, L159 Wilson, J. K., Baumgardner, J., \& Mendillo, M. 1998, Geophys. Res. Lett., 25, 225 